\title{
Determinants of antenatal depression and postnatal depression in Australia
}

Felix Akpojene Ogbo ${ }^{1,2^{*}}$, John Eastwood ${ }^{2,3,4,5,6}$, Alexandra Hendry ${ }^{2,7}$, Bin Jalaludin ${ }^{2,8}$, Kingsley E. Agho', Bryanne Barnett ${ }^{9}$ and Andrew Page ${ }^{1,2}$

\begin{abstract}
Background: Depression is a leading source of morbidity and health loss in Australian women. This study investigates the determinants of antenatal depressive symptoms and postnatal depressive symptoms in an Australian population, including people from culturally and linguistically diverse (CALD) backgrounds.

Method: The study used a retrospective cohort of mothers of all live births in public health facilities in 2014 $(N=17,564)$ within South Western Sydney Local Health District and Sydney Local Health District in New South Wales, Australia. Prevalence of antenatal and postnatal depressive symptoms were estimated for the cohort. Multivariate logistic regression models were conducted to investigate the sociodemographic, psychological and health service determinants of antenatal and postnatal depressive symptoms, measured using the Edinburgh Postnatal Depression Scale (EPDS).

Results: The prevalence of antenatal and postnatal depressive symptoms was $6.2 \%$ and $3.3 \%$ of the cohort, respectively. Significant risk factors for maternal depressive symptoms during pregnancy were, a lack of partner support, history of intimate partner violence, being from the CALD population and low socioeconomic status. Self-reported antenatal depressive symptoms were strongly associated with postnatal depressive symptoms. Risk factors for postnatal depressive symptoms were similar to those for antenatal depressive symptoms, as well as assisted delivery.

Conclusion: Factors relating to demographic and psychosocial disadvantage were associated with subsequent antenatal and postnatal depressive symptoms in New South Wales, Australia. Our study suggests that screening for probable depression and timely referral for expert assessment of at-risk mothers may be an effective strategy to improve maternal mental health outcomes.
\end{abstract}

Keywords: determinants, antenatal, depression, perinatal, postnatal, Australia, pregnancy

\section{Background}

Depression is a major public health issue worldwide and was among the leading source of health loss in terms of disability-adjusted life years among Australian women of reproductive age in 2016 [1, 2]. Maternal depression during pregnancy (antenatal depression) and postnatal depression have both short- and long-term consequences for the mother, the child and family. For the infant, these may include low birth weight, psychological

\footnotetext{
* Correspondence: f.ogbo@westernsyndey.edu.au

${ }^{1}$ Translational Health Research Institute, School of Medicine, Western Sydney University, Campbelltown Campus, Locked Bag 1797, Penrith, NSW 2571, Australia

${ }^{2}$ Ingham Institute for Applied Medical Research, University of New South Wales, 1 Campbell Street, Liverpool, NSW 2170, Australia

Full list of author information is available at the end of the article
}

issues, increased risk of experiencing diarrhoea and suboptimal infant feeding patterns [3-6]. Lower rates of immunisation, and reduced growth and developmental trajectories have also been reported in infants whose mothers had perinatal depression [7]. In addition, stress, poor social interaction and lost productivity have been documented in pregnant women with depression $[8,9]$.

In Australia, recent studies have indicated that maternal depression during pregnancy is associated with adverse perinatal outcomes $[10,11]$. Other Australian studies have reported higher prevalence for antenatal depression, from $6 \%[4,10]$ to $17 \%[12]$ and postnatal depression, from $6 \%$ to $12 \%[4,12,13]$. A study conducted among women living in a socioeconomically disadvantaged community in South Australia indicated that the incidence of antenatal 
depressive symptoms over a seven-month period was $30 \%$, reflecting variation in the burden of perinatal depression in women across the socioeconomic scale [14].

Previous studies conducted in Australia have shown a range of factors associated with antenatal and postnatal depression. Risk factors for antenatal depression include low self-esteem, anxiety, low social support, negative cognitive style, major life events, low income and history of abuse [12, 14], domestic violence during pregnancy and having recently hit someone else in anger [14]. Risk factors for postnatal depression include antenatal depression and history of depression [12], poor health status of the mother, not breastfeeding, difficult financial situation and being born overseas [13]. Furthermore, analysis of data from the beyondblue National Depression Program in Australia found that antenatal depression, a prior history of depression and limited partner support were the strongest risk factors for postnatal depression [15].

The Edinburgh Postnatal Depression Scale (EPDS) is routinely used for pregnant women in many Australian primary health care settings to identify and refer for expert assessment and management, women who may be experiencing or are at risk of perinatal depression. The National Postnatal Depression Program recommends the EPDS as a screening device for perinatal depression, and advocates for ongoing research in perinatal depression to inform policy formulation, and targeted and evidence-based initiatives $[4,16]$. Accordingly, the present study aimed to investigate socioeconomic, psychosocial and health service determinants of maternal depressive symptoms during pregnancy and postnatal depressive symptoms in Australia, including people from culturally and linguistically diverse (CALD) populations.

\section{Methods}

\section{Data source}

The study was based on a retrospective cohort of mothers of all live births $(N=17,564)$ in public health facilities within South Western Sydney Local Health District (SWSLHD) and the Sydney Local Health District (SLHD) in 2014, in the state of New South Wales (NSW), Australia. Antenatal data, routinely collected by qualified midwives, were linked to routinely collected postnatal data (by skilled community health nurses), using individual identifiers. These data were stored in the Information Management \& Technology Division (IM\&TD) database of the health districts. After ethics approvals were obtained, the data were cleaned and coded for analysis.

\section{Study setting}

SWSLHD and SLHD represent approximately $52 \%$ of the Sydney metropolitan region, with a population of over 1,457,100 people. This region of Sydney has one of Australia's most culturally diverse populations [17, 18], with approximately $50 \%$ of mothers born overseas, with mothers from Middle Eastern countries (10\%), South East Asia (8\%) and Southern Asia (8\%) most commonly represented [19]. The health districts provide health services to a socioeconomically diverse population, with some geographic areas in South Western Sydney representing some of the most socioeconomically disadvantaged and advantaged areas in the Sydney metropolitan region [10].

\section{Risk factors}

Study variables included: maternal age (categorised as less than 20 years, $20-34$ years or over 35 years); areabased socioeconomic status (SES, categorised as low, medium or high); partner support (categorised as Yes or No/unsure); culturally and linguistically diverse (CALD, categorised as Yes or No); history of physical intimate partner violence (IPV, categorised as Yes or No); fear of partner/ex-partner (categorised as Yes or No); history of pre-existing antenatal health problems (such as diabetes mellitus and hypertension, categorised as Yes or No); and type of delivery (categorised as normal vaginal, assisted vaginal or caesarean delivery). Sociodemographic, psychological and health service data were collected in the first prenatal clinic visit for the index pregnancy, and information on the type of delivery was collected soon after birth.

Area-based SES was measured using the SocioEconomic Index for Areas (SEIFA), based on the mother's address. SEIFA is a measurement indicator developed by the Australian Bureau of Statistics that ranks areas in Australia according to relative socio-economic advantage and disadvantage [20]. Deciles of SES were categorised into high (top 10\% of the population), medium (middle $80 \%$ of the population) and low (bottom $10 \%$ of the population) groups, consistent with previous publications [10, 19]. CALD is a term used for communities with diverse language, ethnic background, nationality, dress, traditions, food, societal structures, art and religion characteristics [21]. IPV information was collected during antenatal period based on the NSW Routine Domestic Violence Screening policy, where mothers were asked: "Within the last 12 months, have you been hit, slapped or hurt in other ways by your partner or ex-partner (physical IPV)?" or "Are you frightened of your partner or ex-partner (psychological IPV)?"

\section{Outcome variables}

In the present study, maternal depressive symptoms during pregnancy and postnatal depressive symptoms were the main outcomes, measured using the EPDS, which has been validated in the antenatal and postnatal 
periods [22-24]. The EPDS has been recommended as a screening tool for perinatal depression among women in Australia [4]. Midwives collected information on sociodemographic and psychosocial characteristics, and maternal depressive symptoms during pregnancy at the first antenatal care visit. For non-English speaking mothers, the English version of the EPDS was administered through qualified interpreters, certified by the National Accreditation Authority for Translators and Interpreters in Australia. The overall number of antenatal depressive symptoms was calculated to achieve a total score (out of $30)$ and was then coded as a categorical variable (score $<13$ or score $\geq 13$ ), with a score of greater or equal to 13 suggestive of antenatal depressive symptoms [24].

Data on postnatal depressive symptoms were collected within the first six weeks of birth. The total number of postnatal depressive symptoms was also tallied to obtain a total score (out of 30) and was then coded as a categorical variable (score $<13$ or score $\geq 13$ ) to indicate scores suggestive of postnatal depressive symptoms [24]. In the present analysis, the EPDS cut-points used to indicate probable depression were selected based on previously published studies $[10,13,25,26]$ and the NSW government guidelines on improving mental health outcomes for parents and infants [27].

The EPDS is the most common and acceptable screening tool for identifying depressive symptoms in the perinatal period worldwide, with a reported sensitivity of $68-86 \%$ and specificity of $78-96 \%[23,24]$. In Australia, a study that used the EPDS among a sample of 4148 women, reported a sensitivity of $100 \%$ and specificity of $89 \%$ [28]. Studies have reported a positive predictive value (PPV) for clinical depression [23] and a PPV of approximately $70 \%$ with an EPDS score of $>12[24,28,29]$.

\section{Statistical analysis}

Preliminary analyses involved a series of frequencies and cross-tabulations to estimate the prevalence of antenatal and postnatal depressive symptoms by study variables. This was followed by univariate logistic regression analysis to examine all potential risk factors associated with antenatal and postnatal depressive symptoms. Only those variables with $P$-value $<0.1$ in univariate models were then entered into a multivariate model to estimate the factors associated with antenatal and postnatal depressive symptoms. Univariate and multivariate odds ratios (ORs) and their 95\% confidence intervals were reported in the present study.

\section{Missing data}

The study investigated the potential effect of missing data on observed odds ratios in sensitivity analyses, which were conducted on an imputed data set based on the original cohort comprising complete outcome data
(Figs. 1 and 2). Multivariate imputation by chained equation was used to impute missing information on study factors included in the analysis. This approach assumes that data were missing at random and that the characteristics of known participants can be used to calculate the characteristics of participants with missing data [30]. Sensitivity analyses were conducted using the ice command in Stata software (Stata Corp, V.15.0, College Station, TX, USA), based on 25 multiple imputations [31]. Revised odds ratios and corresponding confidence intervals were estimated from the imputed dataset, using the mim command.

\section{Results}

Antenatal depressive symptoms (EPDS $\geq 13$ )

The prevalence of mothers who reported depressive symptoms during pregnancy was $6.2 \%$ in the cohort and was higher among mothers who reported psychological IPV (28\%) or physical IPV (32\%) [Table 1]. The prevalence of ANC depressive symptoms was higher in people from the CALD background (8.0\%) compared to those from the non-CALD group (4.0\%). A lack of partner support ( $\mathrm{OR}=$ 6.1, 95\% CI: 4.6-7.9, $P<0.001)$ and being from the CALD group (OR $=2.0,95 \% \mathrm{CI}: 1.6-2.4, P<0.001)$ were associated with antenatal depressive symptoms. Intimate partner violence was associated with depressive symptoms during pregnancy compared to mothers with no history of IPV (OR $=2.6,95 \%$ CI: 1.6-4.2, $\mathrm{P}<0.001$ for physical IPV and $\mathrm{OR}=4.8,95 \% \mathrm{CI}: 3.0-7.8, \mathrm{P}<0.001$ for psychological IPV). Mothers from high SES group were significantly less likely to experience depressive symptoms during pregnancy compared to mothers from low SES group $(\mathrm{OR}=0.5,95 \%$ CI: 0.3-0.7, $\mathrm{P}<0.001)$ [Table 1].

\section{Postnatal depressive symptoms (EPDS $\geq 13$ )}

The prevalence of postnatal depressive symptoms was $3.3 \%$, and maternal depressive symptoms during pregnancy were strongly associated with postnatal depressive symptoms $(\mathrm{OR}=6.7,95 \% \mathrm{CI}$ : 4.9-9.3, $P<0.001)$ [Table 2]. Compared to mothers from the low SES group, mothers from higher SES group were less likely to experience postnatal depressive symptoms $(\mathrm{OR}=0.7,95 \% \mathrm{CI}$ : $0.5-$ $0.9, P=0.010$ for middle SES group and $\mathrm{OR}=0.5,95 \%$ CI: $0.3-0.8, P=0.025$ for high SES group). The prevalence of postnatal depressive symptoms was higher in mothers from the CALD group (4.2\%), and mothers who identified as being from the CALD group were also more likely to experience postnatal depressive symptoms compared to those, not from the CALD group $(\mathrm{OR}=$ 1.5, 95\%CI 1.1-2.0, $P=0.002$ ). Psychological IPV was strongly associated with postnatal depressive symptoms $(\mathrm{OR}=5.3$, 95\% CI: 2.5-11.0, $P<0.001)$, and mothers who had a caesarean section were more likely to experience postnatal depressive symptoms compared to those 


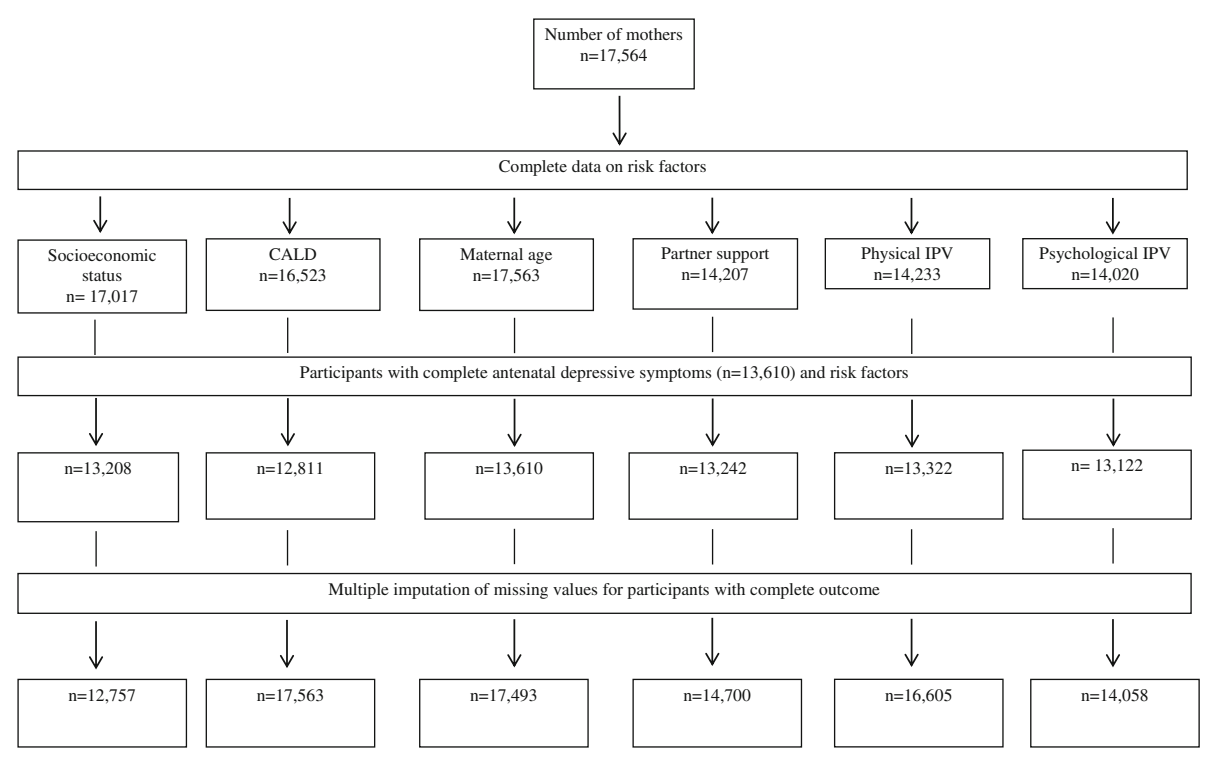

Fig. 1 Flow chart of available data on antenatal depressive symptoms and risk factors in South Western Sydney Local Health District and Sydney Local Health District in New South Wales, Australia (2014)

who had normal vaginal delivery $(\mathrm{OR}=1.7$, 95\% CI: $1.3-$ 2.3, $\mathrm{P}<0.001$ ).

Stratified analyses showed no substantial, significant differences between CALD and non-CALD groups. For example, mothers who experienced physical and psychological IPV were almost equally distributed in both groups (50\% in both groups for physical IPV, and 49.0\% and $51.0 \%$ among non-CALD and CALD population for psychosocial IPV, respectively). The revised odds ratios from sensitivity analyses were not markedly different from the complete case analysis (except for the association between younger maternal age and antenatal depressive symptoms), indicating that missing data did not substantially affect the observed findings.

\section{Discussion}

The prevalence of maternal depressive symptoms during pregnancy was $6.2 \%$, with a higher prevalence observed in mothers with a history of IPV. The analysis showed that a lack of partner support, IPV, being from the

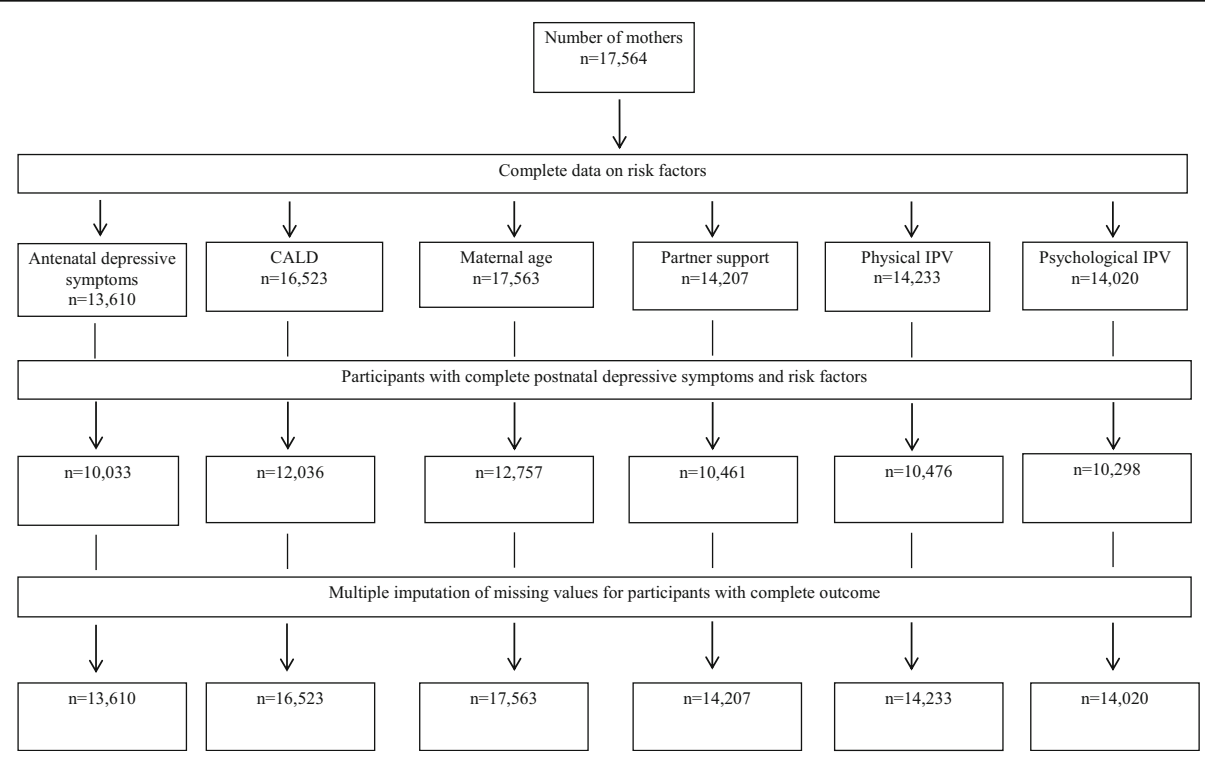

Fig. 2 Flow chart of available data on postnatal depressive symptoms and risk factors in South Western Sydney Local Health District and Sydney Local Health District in New South Wales, Australia (2014) 
Table 1 Study factors and antenatal depressive symptoms of mothers from South Western Sydney and Sydney Local Health Districts in $2014(N=17,564)$

\begin{tabular}{|c|c|c|c|c|c|c|c|c|c|c|c|}
\hline \multirow{2}{*}{ Outcome } & \multirow[b]{2}{*}{ Participants } & \multirow[b]{2}{*}{ Cases } & \multirow[b]{2}{*}{$\%$} & \multicolumn{4}{|c|}{ Complete cases } & \multicolumn{4}{|c|}{ Multiple imputation ${ }^{a}$} \\
\hline & & & & $\begin{array}{l}\text { Unadjusted } \\
\text { OR }(95 \% \mathrm{Cl})\end{array}$ & $P$ value & $\begin{array}{l}\text { Adjusted } \\
\text { OR }(95 \% \mathrm{Cl})\end{array}$ & $P$ value & $\begin{array}{l}\text { Unadjusted } \\
\text { OR }(95 \% \mathrm{Cl})\end{array}$ & $P$ value & $\begin{array}{l}\text { Adjusted } \\
\text { OR }(95 \% \mathrm{Cl})\end{array}$ & $P$ value \\
\hline Antenatal depressive symptoms & 13,610 & 846 & 6.2 & & & & & & & & \\
\hline \multicolumn{12}{|l|}{ Socio-economic status } \\
\hline Low & 5716 & 450 & 7.9 & 1.0 & & 1.0 & & 1.0 & & 1.0 & \\
\hline Medium & 5968 & 321 & 5.4 & $0.6(0.5-0.7)$ & $<0.001$ & $0.8(0.7-1.0)$ & 0.060 & $0.6(0.5-0.7)$ & $<0.001$ & $0.8(0.7-0.9)$ & $<0.001$ \\
\hline High & 1524 & 43 & 2.8 & $0.3(0.2-0.4)$ & $<0.001$ & $0.5(0.3-0.7)$ & 0.001 & $0.3(0.2-0.4)$ & $<0.001$ & $0.5(0.4-0.5)$ & $<0.001$ \\
\hline \multicolumn{12}{|l|}{ CALD group } \\
\hline No & 6173 & 278 & 4.5 & 1.0 & & 1.0 & & 1.0 & & 1.0 & \\
\hline Yes & 6638 & 528 & 8.0 & $1.8(1.5-2.1)$ & $<0.001$ & $2.0(1.6-2.4)$ & $<0.001$ & $1.6(1.6-1.7)$ & $<0.001$ & $1.8(1.7-1.9)$ & $<0.001$ \\
\hline \multicolumn{12}{|l|}{ Supportive Partner } \\
\hline Yes & 12,805 & 666 & 5.2 & 1.0 & & 1.0 & & 1.0 & & 1.00 & \\
\hline No/unsure & 437 & 140 & 32.0 & $8.5(6.9-10.6)$ & $<0.001$ & $6.1(4.6-7.9)$ & $<0.001$ & $8.5(8.2-8.9)$ & $<0.001$ & $6.1(5.9-6.4)$ & $<0.001$ \\
\hline \multicolumn{12}{|l|}{ Antenatal health problems } \\
\hline No & 8573 & 465 & 5.4 & 1.0 & & 1.0 & & 1.0 & & 1.0 & \\
\hline Yes & 3774 & 273 & 7.2 & $1.3(1.1-1.5)$ & $<0.001$ & $1.2(1.0-1.5)$ & 0.003 & $1.3(1.3-1.4)$ & $<0.001$ & $1.2(1.2-1.3)$ & $<0.001$ \\
\hline \multicolumn{12}{|l|}{ Physical intimate partner violence } \\
\hline No & 13,140 & 182 & 5.9 & 1.0 & & 1.0 & & 1.0 & & 1.0 & \\
\hline Yes & 777 & 50 & 27.5 & $6.0(4.3-8.4)$ & $<0.001$ & $2.6(1.6-4.2)$ & $<0.001$ & $6.4(6.1-6.8)$ & $<0.001$ & $3.1(2.9-3.4)$ & $<0.001$ \\
\hline \multicolumn{12}{|c|}{ Psychological intimate partner violence } \\
\hline No & 12,989 & 768 & 5.9 & 1.0 & & 1.0 & & 1.0 & & 1.0 & \\
\hline Yes & 133 & 43 & 32.3 & $7.0(5.2-11.0)$ & $<0.001$ & $4.8(3.0-7.8)$ & $<0.001$ & $8.0(7.6-8.5)$ & $<0.001$ & $4.1(3.8-4.4)$ & $<0.001$ \\
\hline \multicolumn{12}{|l|}{ Maternal age group } \\
\hline 20-34 years & 9382 & 571 & 6.1 & 1.0 & & 1.0 & & 1.0 & & 1.0 & \\
\hline$>35$ years & 3206 & 207 & 6.5 & $1.0(1.1-2.5)$ & 0.452 & $1.0(0.8-1.2)$ & 0.624 & $1.0(1.0-1.1)$ & $<0.001$ & $1.1(1.0-1.1)$ & $<0.001$ \\
\hline$<20$ years & 243 & 24 & 9.9 & $1.7(1.1-2.6)$ & 0.017 & $1.4(0.8-2.5)$ & 0.178 & $1.5(1.4-1.6)$ & $<0.001$ & $1.3(1.2-1.4)$ & $<0.001$ \\
\hline
\end{tabular}

Data on risk factors and antenatal depressive symptoms were collected at the first antenatal clinic visit. Multivariate model adjusted for variables with $\mathrm{p}$-value $<$ 0.1 in univariate analysis. The variables include maternal age, socioeconomic status, CALD group, supportive partner, history of physical intimate partner violence, antenatal health problems and history of fear of partner/ex-partner

CALD culturally and linguistically diverse

${ }^{\text {a }}$ Sensitivity analyses following multiple imputations for missing values

CALD population and low SES group were associated with maternal depressive symptoms during pregnancy. The prevalence of postnatal depression was $3.3 \%$, and the risk factors for postnatal depressive symptoms were assisted delivery (caesarean section), low SES, psychological IPV, being from the CALD population and antenatal depressive symptoms.

The impact of antenatal depression on perinatal outcomes in the study participants has been documented [10] and was consistent with previous reports $[5,11]$. Similarly, most of the previously established risk factors for antenatal depression in other contexts also played a role in predicting maternal depressive symptoms during pregnancy in the current study. A number of the identified risk factors are amenable to initiatives, suggesting that well-targeted interventions and policies may be helpful in reducing the burden of antenatal depression. For example, IPV and a lack of partner support were the strongest risk factors for antenatal depressive symptoms in the present study, consistent with evidence from previous reports [12, 32]. The Australian, State and Territory governments commitment to reducing perinatal depression, IPV and improve partner support are broader initiatives needed to improve the socialemotional and physical health of both mother and infant health [33-35].

In the state of NSW, there are facility- and community-based interventions that aim to identify vulnerable mothers with probable depressive episodes quickly, and referral for expert assessment and 
Ogbo et al. BMC Psychiatry (2018) 18:49

Page 6 of 11

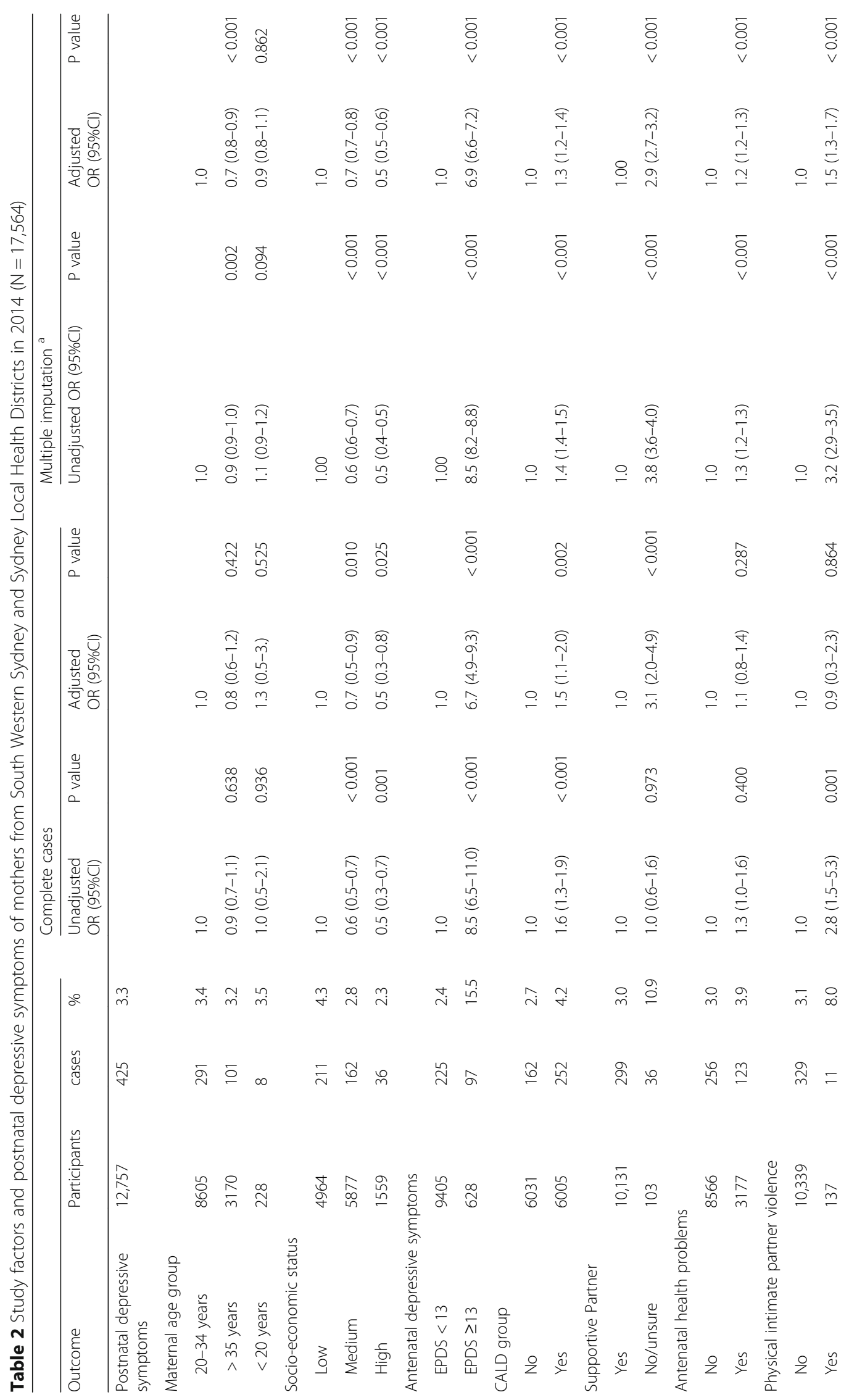




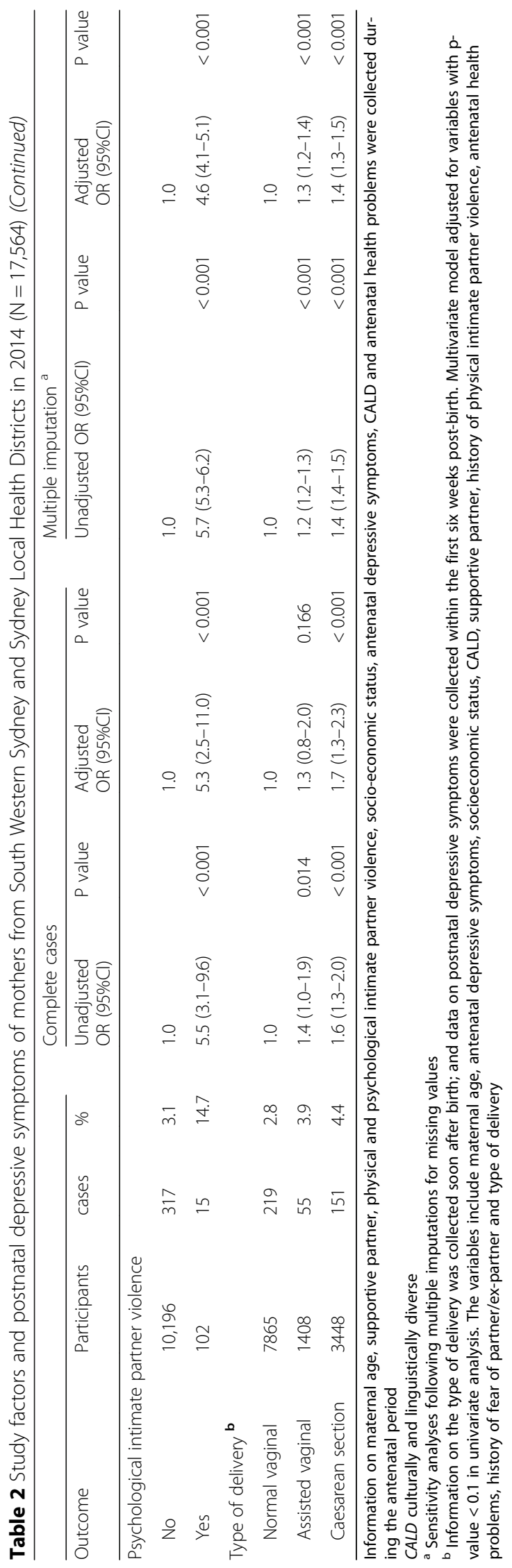


subsequent management [34, 36-38]. While those initiatives are important and reportedly well-received, it is also worth noting that risk factors for antenatal depression are multi-faceted, and would require multidisciplinary and multi-agency intervention approaches that are targeted, collaborative and coordinated to improve health outcomes [39]. More recently, the NSW government has begun moving to a more patient-centred integrated health system, with connected service provision across different providers [40]. Aspects of the strategy have been shown to identify barriers to care and facilitate care for vulnerable families, including those with depressive symptoms $[41,42]$. Scaling up such an approach for at-risk mothers will improve not only maternal and infant health, but also the family's social interaction and long-term productivity.

The study found that the prevalence of antenatal depressive symptoms was similar to a previous national report [4]. This finding underscores the utility of the EPDS, applied in the Australian primary health care setting, to promptly identify women with probable depression and referral for professional assessment. Our study indicated that the proportion of women who reported postnatal depressive symptoms was lower than the national prevalence (19\%) [43] and prior studies from Australia (6.2-10.4\%) $[11,13,44]$. However, a recent systemic review found that depression among Australian women ranged from $2.6 \%$ to $43.9 \%$, highlighting the disparities in mental health burden across the lifespan $[43,45]$.

A plausible explanation for the low prevalence of postnatal depressive symptoms in our study may be due to the period in which the data were collected. The postnatal data were collected within the first six weeks' post-birth, while the national survey was based on the first year of the birth of the child. Additionally, the prevalence of postnatal depressive symptoms may have varied from previous studies because of the impact of the integrated perinatal care initiatives $[36,46]$ and other family-centred services in the region [47]. Formal evaluation of the perinatal care interventions is warranted to determine the extent to which the initiatives have been context-specific.

Mothers from low SES groups and being from the CALD group were at risk of experiencing pre- and/or post-pregnancy depressive symptoms, in line with previous studies [12, 14]. Plausible reasons for why being from the CALD group increases the risk of prenatal and/or postnatal depressive symptoms among mothers have been highlighted in Australia. These include sociocultural barriers (e.g., cultural norms, language and traditional gender roles); structural barriers (e.g., a lack of knowledge of available services and issues with access); service-related barriers (e.g., culturally-inappropriate service models or a perception of such services); domestic violence problems [48], and alcohol and other drugs issues [49]. A number of those barriers may also apply to mothers from low SES groups, as well as issues of limited uptake of health messages associated with mothers from low-income households [50]. Although being from the CALD group is not modifiable or improvement in SES status can be a long-term project; targeted and culturally-appropriate initiatives that are specific to mothers in those populations will improve health outcomes. A report from Australia estimated that maternal perinatal depression cost the Australian economy 171 million dollars in 2012, and the majority of the burden was attributable to lost productivity in the workplace [35]. This report further highlights the fact that routine screening for perinatal depression and subsequent referral for further assessment of vulnerable women is essential to improving health and economic productivity.

The study has a number of policy and pragmatic implications for health professionals, health administrators, researchers and the public. Our study provides risk factors for antenatal and postnatal depressive symptoms in Australia, as well as among people from culturally diverse communities, using routinely collected and context-specific data. Evidence has shown that strong government support is critical to health interventions aimed at identifying and/or treating diseases in the early stages $[4,16]$. The NSW government SAFE START policy framework is one of such support, aimed at providing planned, coordinated and complete psychosocial assessment, depression screening and follow-up care and support for mothers and babies during the perinatal period [36]. Although the SAFE START initiative is still active in NSW, in 2015, the Australian federal government stopped a funding agreement with the states and territories government for a National Perinatal Depression Initiative (NPDI) which provided new parents access to screening and counselling services since the year 2008 [51]. Studies that evaluate the impact of the NPDI and other sub-national policy responses and interventions to perinatal depression may be warranted in Australia to measure where improvements have been made, where opportunities are or where challenges occur. Further studies on the impact and possible referral pathways of moderate perinatal depression may also be needed.

\section{Study limitations and strengths}

The study has a number of limitations. First, the outcome of interest and some of the risk factors (e.g., IPV) were based on self-report, which is a possible source of recall or measurement bias that may have 
underestimated or overestimated the association between the risk factors and outcomes. Second, the study used routinely collected data for the analyses of risk factors for antenatal depressive symptoms, and the establishment of clear temporal relationships cannot be determined for such associations, where study factors data and antenatal depressive information were collected contemporaneously. Nonetheless, the study provides evidence on clear temporal relationships between the study factors and postnatal depressive symptoms. Third, unmeasured covariates such as the impact of social support and family structure may be a limitation of the study. Fourth, our study was unable to differentiate mothers with pre-existing clinical depression from those with first-ever perinatal depressive symptoms. A stratified analysis of preexisting and first-ever perinatal depression would have provided additional information on the health status of mothers. Despite these limitations, we take advantage of the routine and consistently collected maternal and infant data in Sydney, Australia to provide context-specific evidence to inform policy decision-making and initiatives. Also, the observed odds ratios are unlikely to be affected by missing data as we considered the potential bias due to missing data in a sensitivity analysis that imputed missing information.

The EPDS is a screening tool, and may not identify all mothers with depressive episodes, as some mothers with high scores may not have clinical depression, while some mothers with low scores may underestimate, or not wish to report distress. The use of the EPDS as the measure for probable depression diagnosis may be less ideal as an assessment of depressive symptoms in the postnatal period may due to extra postpartum duties associated with the arrival of a newborn. Possible limitations of the EPDS tool and an alternative device were recently highlighted [52], similar to previously published shortcomings $[53,54]$ and alternatives to the EPDS $[55,56]$. Nevertheless, it is important to note that even if a screening tool is error-free, its interpretation (i.e., the assignment of a positive or negative outcome) may still be incorrect [57].

Despite the limitations of the EPDS, it is an extremely valuable tool for researchers to evaluate the impact of preventive programs and for clinicians to promptly identify mothers who may be experiencing or are at risk for perinatal depression [4, 16]. Also, the EPDS is unique and designed for use in the perinatal period as it does not measure somatic symptoms associated with depression, as symptoms like weight change and sleep difficulties are associated with normal pregnancies.

\section{Conclusion}

The prevalence of antenatal depressive symptoms was higher in mothers with a history of IPV. A lack of partner support, IPV, being from the CALD population and low SES group were risk factors for antenatal and postnatal depressive symptoms. Antenatal depressive symptoms were strongly associated with postnatal depressive symptoms. The study suggests that screening for probable depression and referral for expert assessment may help to identify at-risk mothers of perinatal depression promptly, and may create pathways for improved maternal mental health care.

\section{Abbreviations \\ CALD: Culturally and linguistically diverse; EPDS: Edinburgh postnatal depression scale; IPV: Intimate partner violence; NPDI: National perinatal depression initiative; NSW: New South Wales; SES: Socio-economic status; SLHD: Sydney Local Health District; SWSLHD: South Western Sydney Local Health District}

\section{Acknowledgements}

The authors are grateful to all the health professionals in South Western Sydney and Sydney Local Health Districts who spent time entering the data, and also to personnel in the Information Management \& Technology

Division for the time spent on generating the data for this analysis.

\section{Funding}

This study received no specific grant from any funding agency in the public, commercial or not-for-profit sectors.

\section{Availability of data and materials}

The data used for the analysis are accessed in accordance with ethical protocols that only allow unit record information to be released to investigators in the ethics committee submission for study approvals. Please send data requests and queries to South Western Sydney Local Health District Ethics committee. Postal Address: Research and Ethics Office Locked Bag 7103 LIVERPOOL BC NSW 1871 Australia Phone: + 61 (02) 8738 8304; Fax: +61 (02) 8738 8310; email: research.support@sswahs.nsw.gov.au Sydney Local Health District Ethics committee c/- Research Ethics and Governance Office (REGO) Royal Prince Alfred Hospital Missenden Road CAMPERDOWN NSW 2050 Australia Telephone: + 61 (02) 9515 6766; Facsimile: + 61 (02) 95157176

\section{Authors' contributions}

FAO contributed to the conceptualisation of the research idea, performed the analysis, interpreted results, drafted the original manuscript and critically revised the manuscript as submitted. JE conceptualised the study idea, obtained and prepared the data, interpreted results and critically revised the manuscript. AH obtained and prepared the data for analysis. BJ contributed to data preparation, results interpretation and critically revised the manuscript. AP, KA and BB provided guidance on the analysis, interpreted results and critically revised the manuscript. All authors read and approved the final manuscript as submitted.

\section{Ethics approval and consent to participate}

Ethics approvals for this study were obtained from the South Western Sydney Local Health District and the Sydney Local Health District Human Research Ethics Committees. No individuals were contacted for this study. Approval numbers HREC: LNR/11/LPOOL/463; SSA: LNRSSA/11/LPOOL/464 and Project No: 11/276 LNR; Protocol No X12-0164 and LNR/12/RPAH/266.

Consent for publication

Not applicable

Competing interests

The authors declare that they have no competing interests. 


\section{Publisher's Note}

Springer Nature remains neutral with regard to jurisdictional claims in published maps and institutional affiliations.

\begin{abstract}
Author details
${ }^{1}$ Translational Health Research Institute, School of Medicine, Western Sydney University, Campbelltown Campus, Locked Bag 1797, Penrith, NSW 2571, Australia. ${ }^{2}$ Ingham Institute for Applied Medical Research, University of New South Wales, 1 Campbell Street, Liverpool, NSW 2170, Australia. ${ }^{3}$ School of Women's and Children's Health, University of New South Wales, Kensington, Sydney, NSW 2052, Australia. ${ }^{4}$ Menzies Centre for Health Policy, Charles Perkins Centre, School of Public Health, Sydney University, Sydney, NSW 2006, Australia. ${ }^{5}$ School of Public Health, Griffith University, Gold Coast, QLD 4222, Australia. ${ }^{6}$ Department of Community Paediatrics, Sydney Local Health District, Croydon Community Health Centre, 24 Liverpool Rd, Croydon, NSW 2132, Australia. ${ }^{7}$ National Centre for Immunisation Research and Surveillance, The Children's Hospital at Westmead, Locked Bag 4001, Westmead, NSW 2145, Australia. ${ }^{8}$ Healthy People and Places Unit, South Western Sydney Local Health District, Liverpool, NSW, Australia. ${ }^{9}$ St John of God Health Care, Blacktown, NSW, Australia.
\end{abstract}

\section{Received: 14 August 2017 Accepted: 8 January 2018}

Published online: 20 February 2018

\section{References}

1. Institute for Health Metrics and Evaluation. GBD Compare Seattle, WA, USA: IHME, University of Washington; 2016 [November 22 2017]. Available from: http://vizhub.healthdata.org/gbd-compare

2. Hay IS, Abajobir AA, Abate KH, Abbafati C, Abbas KM, Abd-Allah F, et al. Global, regional, and national disability-adjusted life-years (DALYs) for 333 diseases and injuries and healthy life expectancy (HALE) for 195 countries and territories, 1990-2016: a systematic analysis for the global burden of disease study 2016. Lancet. 2017;390(10100):1260-344.

3. Dias CC, Figueiredo B. Breastfeeding and Depression: a systematic review of the literature. J Affect Disord. 2015;171:142-54

4. beyondblue National Postnatal Depression Program. The beyondblue National Postnatal Depression Program Prevention and Early Intervention 2001-2005. 2005.

5. Grigoriadis S, EH VP, Mamisashvili L, Tomlinson G, Dennis C-L, Koren G, et al. the impact of maternal depression during pregnancy on perinatal outcomes: a systematic review and meta-analysis. The Journal of clinical psychiatry. 2013;74(4):321-41.

6. Gentile S. Untreated depression during pregnancy: short-and long-term effects in offspring. A systematic review. Neuroscience. 2015

7. Rahman A, lqbal Z, Bunn J, Lovel H, Harrington R. Impact of maternal depression on infant nutritional status and illness: a cohort study. Arch Gen Psychiatry. 2004;61(9):946-52

8. Zuckerman B, Amaro H, Bauchner H, Cabral H. Depressive symptoms during pregnancy: relationship to poor health behaviors. Am J Obstet Gynecol. 1989:160(5):1107-11.

9. Gotlib IH, Hammen CL. Handbook of depression. New York, USA: Guilford Press; 2008

10. Eastwood J, Ogbo FA, Hendry a, Noble J. page a, early years research group. The impact of antenatal depression on perinatal outcomes in Australian women. PLoS One. 2017;12(1):e0169907. https://doi.org/10.1371/journal. pone.0169907

11. Navaratne P, Foo XY, Kumar S. Impact of a high Edinburgh postnatal depression scale score on obstetric and perinatal outcomes. Sci Rep. 2016;6

12. Leigh B, Milgrom J. Risk Factors for antenatal depression, postnatal depression and parenting stress. BMC psychiatry. 2008;8(1):1.

13. Eastwood JG, Phung H, Barnett B. Postnatal depression and sociodemographic risk: factors associated with Edinburgh depression scale scores in a metropolitan area of new South Wales, Australia. Australian \& New Zealand Journal of Psychiatry. 2011;45(12):1040-6.

14. Edwards B, Galletly C, Semmler-Booth T, Dekker G. Antenatal psychosocial risk factors and depression among women living in socioeconomically disadvantaged suburbs in Adelaide, South Australia. Australian \& New Zealand Journal of Psychiatry. 2008:42(1):45-50.

15. Milgrom J, Gemmill AW, Bilszta JL, Hayes B, Barnett B, Brooks J, et al. Antenatal risk factors for postnatal depression: a large prospective study. J Affect Disord. 2008;108(1):147-57.
16. beyondblue. Clinical practice guidelines for depression and related disorders - anxiety, bipolar disorder and puerperal psychosis - in the perinatal period. A guideline for primary care health professionals. Melbourne, Australia: beyondblue, 2011.

17. South Western Sydney Local Health District. Research Strategy for South Western Sydney Local Health District 2012-2021. Sydney, Australia: South Western Sydney Local Health District; 2012.

18. Sydney Local Health District. Planning online: Sydney local Health District; 2016 [22 June 2016]. Available from: https://www.slhd.nsw.gov.au/planning/ profiles.html.

19. Ogbo FA, Eastwood J, Page A, Arora A, McKenzie A, Jalaludin B, et al. prevalence and determinants of cessation of exclusive breastfeeding in the early postnatal period in Sydney, Australia. Int Breastfeed J. 2017;12(1):16.

20. Australian Bureau of Statistics. Technical Paper: Socio-Economic Indexes for Areas (SEIFA) 2011, 2013. Commonwealth of Australia: Canberra.

21. Australian Bureau of Statistics. Culturally and linguistically diversity (CALD) characteristics 2016 [cited 201718 January ]. Available from: http://www. abs.gov.au/ausstats/abs@.nsf/Lookup/by\%20Subject/4529.0.00.003 2014 Main\%20Features

Cultural\%20and\%20Linguistic\%20Diversity\%20(CALD)\%20Characteristics 13.

22. Murray D, Cox JL. Screening for depression during pregnancy with the Edinburgh depression scale (EDDS). Journal of reproductive and infant psychology. 1990;8(2):99-107.

23. Murray L, Carothers AD. The validation of the Edinburgh post-natal depression scale on a community sample. Br J Psychiatry. 1990;157(2):288-90.

24. Cox JL, Holden JM, Sagovsky R. Detection of postnatal depression. Development of the 10-item Edinburgh postnatal depression scale. Br J Psychiatry. 1987;150(6):782-6.

25. Hannah P, Adams D, Lee A, Glover V, Sandler M. Links Between early postpartum mood and post-natal depression. Br J Psychiatry. 1992;160(6):777-80.

26. Kohlhoff J, Hickinbotham R, Knox C, Roach V, Barnett Am B. Antenatal psychosocial assessment and depression screening in a private hospital. Aust N Z J Obstet Gynaecol. 2016;56(2):173-8.

27. NSW Department of Health. NSW Health/Families NSW Supporting Families Early Package - SAFE START Guidelines: Improving Mental Health Outcomes for Parents \& Infants. In: Health Mo, editor. Sydney NSW Health 2010. p. 9-10.

28. Boyce P, Stubbs J, Todd A. The Edinburgh postnatal depression scale: validation for an Australian sample. Aust N Z J Psychiatry. 1993;27(3):472-6.

29. Leverton T, Elliott S. Is the EPDS a magic wand?: 1. A comparison of the Edinburgh Postnatal Depression Scale and health visitor report as predictors of diagnosis on the Present State Examination. 2000.

30. Sterne JA, White IR, Carlin JB, Spratt M, Royston P, Kenward MG, et al. Multiple imputation for missing data in epidemiological and clinical research: potential and pitfalls. BMJ. 2009;338:b2393.

31. Spratt M, Carpenter J, Sterne JA, Carlin JB, Heron J, Henderson J, et al. Strategies for multiple imputation in longitudinal studies. Am J Epidemiol. 2010;172(4):478-87.

32. Biaggi A, Conroy S, Pawlby S, Pariante CM. Identifying the women at risk of antenatal anxiety and depression: a systematic review. J Affect Disord. 2016; 191:62-77.

33. Australian Government Department of Social Services. The National Plan to Reduce Violence against Women and their Children 2010-2022 (the National Plan). In: Department of Social Services, editor. Canberra 2011.

34. Australian Department of Human Services. Family and Domestic Violence Strategy 2016-2019. Can Underwrit. 2016;

35. Deloitte Access Economics. The cost of perinatal depression in Australia. Canberra, ACT: Deloitte, 2012

36. New South Wales Ministry of Health. SAFE START Strategic Policy. In: Mental Health and Drug and Alcohol Office, editor. Sydney, Australia: NSW Health; 2010.

37. NSW Ministry of Health. Violence Prevention and Response Unit. Domestic Violence Routine Screening Program: Snapshot Report 9. 2012.

38. NSW Health. SAFE START guidelines: improving mental health outcomes for parents and infants. NSW Health: Sydney, Australia; 2010.

39. Katz I. Community interventions for vulnerable children and families: Participation and power. Communities, Children and Families Australia. 2007;3(1):19.

40. New South Wales Ministry of Health. NSW Integrated Care Online: NSW Health; 2017 [cited 201714 August]. Available from: http://www.health.nsw. gov.au/integratedcare/Pages/default.aspx.

41. Eastwood J, Miller E. Healthy homes and Neighbourhoods integrated care initiative 2016 [February 2017]. Available from: http://www.health.nsw.gov. au/wohp/Documents/mc7-miller-eastwood.pdf. 
42. Northern NSW Local Health District. NNSW integrated care strategy - status report may 2016. New South Wales: Northern NSW Local Health District; 2016.

43. Australian Institute of Health and Welfare. Experience of perinatal depression: data from the 2010 Australian National Infant Feeding Survey. Canberra: AlHW, 2012 Contract No.: PHE 161.

44. Eastwood JG, Jalaludin BB, Kemp LA, Phung HN, Barnett BE. Relationship of postnatal depressive symptoms to infant temperament, maternal expectations, social support and other potential risk factors: findings from a large Australian cross-sectional study. BMC pregnancy and childbirth. 2012;12(1):148.

45. Rich JL, Byrne JM, Curryer C, Byles JE, Loxton D. Prevalence and correlates of depression among Australian women: a systematic literature review, January 1999-January 2010. BMC research notes. 2013;6(1):424.

46. South Western Sydney Local Health District. SWSLHD mental health service: a clinical stream of south western Sydney local Health District. Sydney: SWSLHD; 2013.

47. Karitane. Mental Health Services: Karitane; 2017 [cited 201714 August]. Available from: https://karitane.com.au/page/our-services/mental-healthservices.

48. Sawrikar P, Katz I. Enhancing family and relationship service accessibility and delivery to culturally and linguistically diverse families in Australia (Vol. 3/ 2008). Australian Institute of Family Studies, AFRC Issues, Australian Family Relationships Clearinghouse: Melbourne; 2008.

49. Browne J, Renzaho A. Prevention Of alcohol and other drug problems in culturally and linguistically diverse communities. Prevention. research quarterly. 2010;13:1-24.

50. Bowes J, Grace R. Review of early childhood parenting, education and health intervention programs for indigenous children and families in Australia. Canberra: Australian Institute of Health and Welfare \& Melbourne: Australian Institute of Family Studies. 2014;

51. David Lewis. National Perinatal Depression Initiative loses federal funding; states, territories 'hard-pressed' to cover cuts Online: ABC; 2015 [cited 2017 31 July ]. Available from: http://www.abc.net.au/news/2015-06-19/ government-pulls-funding-from-perinatal-depression-program/6560266.

52. Matthey S, Agostini F. Using the Edinburgh postnatal depression scale for women and men-some cautionary thoughts. Archives of women's mental health. 2017;20(2):345-54.

53. Cox J, Holden J. Perinatal mental health: a guide to the Edinburgh postnatal depression scale (EPDS): Royal College of psychiatrists; 2003.

54. Allison KC, Wenzel A, Kleiman K, Sarwer DB. Development of a brief measure of postpartum distress. J Women's Health. 2011;20(4):617-23.

55. Somerville S, Byrne SL, Dedman K, Hagan R, coo S, Oxnam E, et al. detecting the severity of perinatal anxiety with the perinatal anxiety screening scale (PASS). J Affect Disord. 2015;186:18-25.

56. Brodey BB, Goodman SH, Baldasaro RE, Brooks-DeWeese A, Wilson ME, Brodey IS, et al. Development of the perinatal depression inventory (PDI)-14 using item response theory: a comparison of the BDI-II, EPDS, PDI, and PHQ9. Archives of women's mental health. 2016;19(2):307-16

57. Rothman K, Greenland S, Lash TL. Modern epidemiology. Philadelphia, USA: Lippincott Williams \& Wilkins; 2008.

\section{Submit your next manuscript to BioMed Central and we will help you at every step:}

- We accept pre-submission inquiries

- Our selector tool helps you to find the most relevant journal

- We provide round the clock customer support

- Convenient online submission

- Thorough peer review

- Inclusion in PubMed and all major indexing services

- Maximum visibility for your research

Submit your manuscript at www.biomedcentral.com/submit 